\title{
On Strongly Nonlinear Poincaré Boundary Value Problems for Harmonic Functions
}

\author{
L. v. WOLFERSDORF
}

Es wird eine Klasse stark linearer Poincaré-Probleme für harmonische Funktionen im Einheitskŕeis durch Zurückführung auf ein neues System von Integralgleichungen untersucht, a uf das der Schaudersche Fixpunktsatz angewendet wird. Für verschiedene Spezialfälle werden konkrete Existenzaussagen' gemacht, insbesondere wird der quasilineare Fall im Detail behandelt.

Исслепуется класс сильно нелинейых задач ГІуанкаре для гармонических функций в единичном круге. Задачи сволятся к новой системе интегральных уравнений, к котород применяетсл, теорема IIаудера о неподвижных точках. Для разных частных случаев даются коікретные теоремы существования. В частности, подробно рассматривается квазијинейный случай.

A class of : strongly nonlinear Poincaré problems for harmonic functions in the unit disk is studied by reducing them to a new integral equation system to which Schauder's fixed point theorem is applied. Specific existence results are given for several special cases; in particular the quasilinear. case is dealt with in detail.

\section{Introduction}

'The Poincaré boundary value problem is a basic problem of the theory of harmonic functions posed by $\mathrm{H}$. Poncart in his investigation. of the mathematical theory of tides in 1910. The plane linear problem of this type containing the problem of oblique derivative as a special case is now fully investigated (cf. [13]).

Existence theorems for nonlinear generalizations of the Poincare problem are derived by W. Pogonzelski [14], J. Wolska-Bochenek $[20,21]$, and M. Schleiff [16] in the case of a linear main part of a boundary operator of Steklov or Poincaré type and a strong nonlinearity in the tangential (and also the normal) derivative of the unknown function $u$ and in $u$ itself satisfying a Hölder-Lipschit $\%$ condition with sufficiently small constant. Corresponding problems in sufficiently small neighborhood of the Neumann and Steklov boundary value problem of potential theory are already investigated by K. MARUHN [12], too. On the other hand there are global existence assertions for this problem with linear main part of oblique derivative or Poincaré type and nonlinearities in the unknown function $u$ alone given by M. Schleiff [16] again and in more general form by $H$. Amann $[1,2]$, F. InkmanN [6], F. Rothe [15], and P. Wildenauer [17].

More intensively, because of its importance in physics, the special case, where the linear main part is the normal derivative of $u$ and the nonlinearity depends only on $u$, has been investigated beginning with the classical paper by T. Carleman [3]. In this context we only mention the papers by K. KLINGELHöFER [7-11], where the method of Hammerstein integral equations is used for proving the existence of solutions for diverse types of such problems and the paper of J. M. Cuserng [4], where a corresponding eigenvalue problem is studied. 
In this paper we make an attempt to investigate nonlinear Poincaré,problems for harmonic functions in the unit disk by another integral equation method developed in our paper [18] for the nonlinear Riemann-Hilbert problem of analytic functions. 'This method works with the differentiated boundary condition on the circumference and reduces the problem to an integral equation system of the type of Villat's equation in the theory of jets. To this system the classical Schauder fixed point theorem is applied.' As a result we obtain existence theorems for some classes of strong nonlinearities in $u$ and the, tangential derivative of $u$ satisfying a constraint on the oscillation of the ascent with respect to the tangential derivative of $u$ and depending in some sense weakly on the function $u$ itself.

\section{Statement of problem}

Let $G: .|z|<1$ be the unit, disk in the complex $z$ plane with boundary $\Gamma:|t|=1$, $t=e^{i s}(-\pi \leqq s \leqq \pi)$. We deal with the following nonlinear Poincaré problem:

Problem P: Find a regular harmonic function $u(z), z=x+i y$, in $G$ which has continuous partial derivatives in $\bar{G}=G+\Gamma$, i.e. $u(z) \in C^{1}(\bar{G})$, and satisfies the boundary condition

$$
\partial u / \partial r+\Phi\left(s, u\left(e^{i s}\right), \partial u / \partial s\right)=f(s) \text { on } \dot{\Gamma}
$$

where $r$ is the polar radius.

The following basic Assumption A on the data is made.

(i) $\Phi(s, u, \omega)$ is a real-valued continuous function on $[-\pi, \pi] \times \mathbf{R} \times \mathbf{R}$ which is $2 \pi$-periodic in $s$ and possesses a continuous partial derivative $\Phi_{\omega}$ and partial derivatives $\Phi_{s}$ and $\Phi_{u}$ satisfying the Carathéodory conditions and estimations of the form

$$
\begin{array}{ll}
\left|\Phi_{s}(s, u, \omega)\right| \leqq E(s) \in L_{e}(\Gamma), & \varrho>1, \\
\left|\Phi_{u}(s, u, \omega)\right| \leqq G(s) \in L_{\varrho}(\Gamma), & \varrho>1,
\end{array}
$$

for $u, \omega$ from bounded intervals of $\mathbf{R}$.

(ii) $f(s)$ is a real-valued absolutely continuous $2 \pi$-periodic function on $[-\pi, \pi]$ - possessing a derivative $f^{\prime}(s) \dot{\epsilon} L_{e}(\Gamma), \varrho>1$.

Under these assumptions we can differentiate the boundary condition (1) with respect to $s$ obtaining the condition

$$
u_{r s}+\Phi_{s}\left(s, u, u_{s}\right)+\Phi_{u}\left(s, u, u_{s}\right) \cdot u_{s}+\Phi_{\omega}\left(s, u, u_{s}\right) \cdot u_{s s}=f^{\prime}(s) \text { a.e. on } \Gamma
$$

where derivatives of $u$ are now denoted by subscripts, too. The boundary condition $(1)$ is equivalent to the condition (4) together with the integral condition

$$
\int_{-\pi}^{\pi} \Phi\left(s, u\left(\mathrm{e}^{i \varepsilon}\right), u_{s}\right) d s=\int_{-\pi}^{\pi} f(s) d s
$$

following from (1) by integration over $\Gamma$.

'We introduce the holomorphic functions in $G$

$$
\begin{aligned}
& w(z)=u(z)+i v(z) ; \quad W(z)=U(z)+i V(z)=z w^{\prime}(z)=r u_{r}-i u_{s}, \\
& X(z)=\dot{\varphi}(z)+i \psi(z)=z W^{\prime}(z)=r U_{s}-i U_{s}=V_{s}+i r V_{r}
\end{aligned}
$$


with

$$
X(t)=\varphi(t)+\dot{i \psi}(t)=-\dot{u_{s s}}-i u_{r s} \text { on . } T .
$$

Then (4) takes the form of a Riemann-Hilbert condition for $/ X(z)$ :

where

$$
\operatorname{Re}\left[\left(A^{\prime}(s)-i\right) \dot{X}(t)\right] \equiv A(s) \dot{\varphi}(t)+\psi(t)=\bar{g}(s) \text { a.e. on } \Gamma \text {, }
$$

$$
\begin{aligned}
& \dot{A}(s)=\Phi_{\omega}\left(s, u, u_{s}\right), \\
& g(s)=\Phi_{s}\left(s, u, u_{s}\right)+\Phi_{u}\left(s, \dot{u}, u_{s}\right) \cdot u_{s}-f^{\prime}(s)
\end{aligned}
$$

The solution $X(z)$ of the Riemann-Hilbert problem:(8) has to fulfil the additional condition

$$
\bar{X}(0)=0 \quad \text { in }{ }^{\prime} z=0
$$

From $X(z)$ the boundary values $u\left(e^{i g}\right)$ of the sought function $u(z)$ follow by the relations.

$$
\begin{aligned}
& u\left(\mathrm{e}^{i s}\right)=\int_{0}^{s} u_{s}\left(\mathrm{e}^{i \sigma}\right) d \sigma+k, \\
& u_{s}\left(\mathrm{e}^{i s}\right)=-\int_{0}^{s} \varphi\left(\mathrm{e}^{i \sigma}\right) d \sigma+k_{1}
\end{aligned}
$$

where

$$
k_{1}=\frac{1}{2 \pi} \int_{-\pi}^{\pi} \int_{0}^{s} \varphi\left(\mathrm{e}^{i \sigma}\right) d \sigma
$$

and the constant $k=u(1)$ has to be determined in fulfilling the integral condition (5).

The harmonic function $u(z)$ itself is then given by the' Poisson integral of the - boundary values $u\left(\mathrm{e}^{i s}\right)$. Thus, Problem $\mathrm{P}$ is equivalent to the relations (12) with

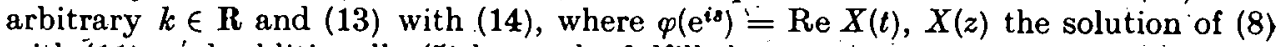
with (11), and additionally (5) has to be fulfilled.

\section{§ 2. Reduction to a fixed point problem}

For prescribed continuous function $A(s)$ and function $g(s) \in L_{e}(\Gamma), \varrho>1$, the Riemann-Hilbert problem (8) with (11) has a unique solution $X(z)$ with $\varphi\left(e^{i 8}\right)=\operatorname{Re} X(t)$ on $\Gamma$ given by. (cf. [5: $\S 29]$ )

$$
\varphi\left(\mathrm{e}^{\dot{i}}\right)=\alpha(s) h(s)+\beta(s) \mathrm{e}^{-H(\mu)(s)} H\left\{\mathrm{e}^{H(\mu)} h\right\}(s),
$$

where

$$
\begin{aligned}
& \alpha(s)=A(\dot{s}) / \sqrt{1+A^{2}(s)}, \quad \beta(s)=i / \sqrt{1+A^{2}(s)}, \\
& \mu(s)=\arctan A(s), \quad h(s)=\dot{g}(s) / \sqrt{1+A^{2}(s)},
\end{aligned}
$$

and $H$ denotes the Hilbert transform

$$
(\dot{H} v)(s)=\frac{1}{2 \pi} \int_{-\pi}^{\pi} v(\sigma) \cot \frac{\sigma-s}{2} d \sigma .
$$


This solution exists if and only if $g(s)$ satisfies the orthogonality condition

$$
\int_{-\pi}^{\pi} \gamma(s) g(s) d s=0
$$

with the nonnegative function

$$
\gamma(s)=\mathrm{e}^{H(\mu)(s)} / \sqrt{1+A^{2}(s)} .
$$

In virtue of a known theorem of A. ZYGMUND (ct. [22]) the function $\mathrm{e}^{H(\mu)(s)}$ and therefore $\gamma(s)$ is sumniable to any power.

For given continuous functions $\xi(s), \eta(s)$ on $\Gamma$ with $\int_{-\pi}^{\pi} \eta(s) d s=0$, i.e. $\xi \in C(\Gamma)$, $\eta \in C_{0}(\Gamma)$, we introduce the auxiliary problem $\mathbf{P}_{\xi, \eta}$ characterized by the boundary condition

$$
A(s, \xi, \eta) \varphi(t)+\dot{\psi}(\ell)=g_{0}(s, \xi, \eta) \text { a.e. on } \Gamma
$$

and the additional condition (11) for $X(z)=\varphi(z)+i \psi(z)$, where

$$
\begin{aligned}
& A(s, \xi, \eta)=\Phi_{\omega}(s, \xi, \eta), \quad \\
& g_{0}(s, \xi, \eta)=g(s, \xi, \eta)-m(s, \xi, \eta)
\end{aligned}
$$

with

$$
\begin{aligned}
& g(s, \xi ; \eta)=\Phi_{s}(s, \xi, \eta)+\Phi_{u}(s, \xi, \eta)^{\prime} \eta-f^{\prime}(s) \\
& m(s, \xi ; \eta)=m_{0}[\xi, \eta] \cdot[\gamma(s, \xi, \eta)]^{\sigma-1}, \quad 1 / \varrho+1 / \sigma=1, \\
& m_{0}[\xi, \eta]=\int_{-\pi}^{\pi} g(s, \xi, \eta) \gamma(s, \xi, \eta) d s / \int_{-\pi}^{\pi} \gamma^{o}(s, \xi, \eta) d s
\end{aligned}
$$

and

$$
\begin{aligned}
& \gamma(s, \xi, \eta)=\mathrm{e}^{H\left(\mu(\cdot, \xi, \eta) \mid(s) / \sqrt{1+A^{2}(s, \xi, \eta)}\right.} \\
& \mu(s, \xi, \eta)=\arctan A(s, \xi, \eta) .
\end{aligned}
$$

The auxiliary problem $\dot{P}_{\xi, \eta}$ has'a unique solution $X(z)$. According to (12), (13), the corresponding boundary values $u(s)=u\left(\mathrm{e}^{i s}\right)$ of $u(z)$ and $u_{1}(s)$ of $u_{s}(z)$ are given by the expressions

$$
\begin{aligned}
& u(s) \doteq N_{1}[\eta](s)=\int_{0}^{s} \eta(\sigma) d \sigma+k, \\
& u_{1}(s)=N_{2}[\xi, \eta](s)=-\int_{0}^{s} \varphi(\sigma, \xi, \eta) d \sigma+k_{1},
\end{aligned}
$$

where

$$
k_{1}=\frac{1}{2 \pi} \int_{-\pi}^{\pi} \int_{0}^{s} \varphi(\sigma, \xi, \eta) d \sigma d s
$$

and $k=k[\eta]$ has to be determined in fulfilling the relation

$$
\int_{-\pi}^{\pi} \Phi\left(s, \int_{0}^{s} \eta(\sigma) d \sigma+k, \eta(s)\right) d s=\int_{-\pi}^{\pi} f(s) d s .
$$


The function $\varphi(s, \xi, \eta)=\varphi\left(\mathrm{e}^{i s}, \xi, \eta\right)$ is defined by (15) with (16), (17), where $A$ is replaced by $A(s, \xi, \eta)$ from (22) and $g$ by $g_{0}(s, \xi, \eta)$ from (23). By Assumption $A$ the function $A(s, \xi, \eta)$ is continuous on $\Gamma$ and the function $g_{0}(s, \xi, \eta) \in L_{e}(\Gamma)$ for any pair of continuous functions $\xi(s), \eta(s)$. Moreover, ' $g_{0}$ fulfils the orthogonality condition (19) by construction.

Any fixed point $\left\{u, u_{1}\right\} \in C(\Gamma) \times C_{0}(\Gamma)$ of the operator $N=\left\{N_{1}, N_{2}\right\}$ yields a solution $u(z)$ of Problem $\mathrm{P}$ with boundary values $u(s)$. For, $u=\xi$ and $u_{1}=\eta$ in (29), (30) at first implies $u_{1}=u^{\prime}(s)$ and further leads to the equation (4) with an additional term $m_{0}\left[u, u_{s}\right] \cdot\left[\gamma\left(s, u, u_{s}\right)\right]^{\sigma-1}$ in the right-hand side which is seen to be zero by integrating the equation over $\Gamma$. Also, from (30) follows that $u_{1}=u_{s} \mid \Gamma \in C_{0}(\Gamma)$ indeed is a Hölder continuous function such that also the boundary values of the normal derivative $u$, of $u$ are (Hölder-) continuous and $u(z) \in C^{1}(\bar{G})$. Thus, Problem $\mathrm{P}$ is reduced to the determination of fixed points for the integral operators $(29),(30)$ with (31), (32).

\section{\$ 3. Existence theorem}

We consider the operator $N=\left\{N_{1}, N_{2}\right\}$ on the convex compact subset $\mathfrak{A}=\mathfrak{R}_{1} \times \mathfrak{R}_{2}$ of the space $C(\Gamma) \times C_{0}(\Gamma)$ with

$$
\begin{aligned}
& \Re_{1}=\left\{\xi \in C(\Gamma):|\xi(s)| \leqq P,\left|\xi\left(s_{1}\right)-\xi\left(s_{2}\right)\right| \leqq P_{0}\left|s_{1}-s_{2}\right|\right\}, \\
& \Re_{2}=\left\{\eta \in C_{0}(\Gamma):|\eta(s)| \leqq R, \quad\left|\eta\left(s_{1}\right)-\eta\left(s_{2}\right)\right| \leqq R_{0}\left|s_{1}-s_{2}\right|^{\prime}\right\}
\end{aligned}
$$

for any $s, \dot{s}_{1}, s_{2} \in[-\pi, \pi]$, where $\lambda=1 / q ; q$ the conjugate exponent to $p, 1<p<\varrho$, and $P, P_{0}, \dot{R}, R_{0}$ are fixed positive real numbers to be specified later.

Further, we make the Assumption B that.for all $\eta \in \Omega_{2}$ the equation (32) for $k \in \mathbf{R}$ has a root $k=k[\eta]$ which depends continuously on $\eta$ and is uniformly bounded with respect to $\eta \in \mathbb{R}_{2}$ :

$$
|k[\eta]| \leqq K \text {, for any } \eta \in \mathfrak{R}_{2} \text { with } K=K\left(R, R_{0}\right) .
$$

This is especially fulfilled if the (continuous) function $\Phi(s, u, \omega)$ is strictly monotone with respect to $u$ for any $s \in[-\pi, \pi], \omega \in \mathbf{R}$, there exist the limits

$$
\lim _{u \rightarrow \pm \infty} \Phi(s, u, \omega) \doteq \Phi_{ \pm}(s) \in C(\Gamma) \text { uniformly in } s \in[-\pi, \pi], \quad \omega \in \mathbf{R}, 1
$$

and $\int_{-\pi}^{\pi} f(s) d s$, lies between the values $\Phi_{ \pm}=\int_{-\pi}^{\pi} \Phi_{ \pm}(s) d s$.

Let us now estimate $u=N_{1}[\eta]$ and $u_{1}=N_{2}[\xi, \eta]$ for $\xi \in \mathfrak{R}_{1}, \eta \in \mathfrak{R}_{2}^{\circ}$. Obviously,

$$
|u(s)| \leqq 2 \pi R+K\left(R, R_{0}\right), \quad \therefore\left|u\left(s_{1}\right)-u\left(s_{2}\right)\right| \leqq R\left|s_{1}-s_{2}\right|
$$

for any $s, s_{1}, \dot{s}_{2} \in[-\pi, \pi]$. Further, for the $L_{Q}$ norm of the function $g_{0}$ there holds

$$
\left\|g_{0}\right\|_{e} \leqq 2\|g\|_{e} \leqq 2\left\{M+\nu_{P, R}+R \beta_{P, R}\right\},
$$

where $M=\left\|f^{\prime}\right\|_{\varrho}$,

$$
\begin{aligned}
& v_{P, R}=\sup \left\|\Phi_{s}(s, u, \omega)\right\|_{\ell}<\infty, \\
& \beta_{P, R}=\sup \left\|\Phi_{u}(s, u, \omega)\right\|_{e}<\infty,
\end{aligned}
$$

the suprema are taken over $s \in[-\pi, \pi],|u| \leqq P ;|\omega| \leqq R$. Finally, applying the triangel inequality, the elementary inequalities, $|\beta(s)| \leqq 1$ and $|\alpha(s) \beta(s)| \leqq 1 / 2$, 
Hölder's inequality and the boundedness of the Hilbert transform in Lebesgue spaces, for the $L_{p}$ norm of the function $\varphi$ we obtain the estimate

$$
\begin{aligned}
-\|\varphi\|_{p} & \leqq(1 / 2)\left\|g_{0}\right\|_{p}+A_{r}\left\|\mathrm{e}^{-H(\mu)}\right\|_{x} \cdot\left\|\mathrm{e}^{H(\mu)} g_{0}\right\|_{r} \\
& \leqq\left\|g_{0}\right\|_{e}\left\{(1 / 2)(2 \pi)^{2 / x}+A_{r}\left\|\mathrm{e}^{-H(\mu)}\right\|_{x} \cdot\left\|\mathrm{e}^{H(\mu)}\right\|_{x}\right\},
\end{aligned}
$$

where $x=2 p \varrho /[\varrho-p], r=x[\varrho-p] /[\varrho+p]$, and $A_{r}$ is the M. Riesz constant, the norm of the Hilbert transform in $\dot{L}_{r}(\Gamma)$.

We make the Assumption C that.

$$
\text { - } 2 \gamma_{P, \ddot{R}}=\sup \mu(s, u, \omega)-\inf \mu(s, u, \omega)<\pi / x
$$

for the oscillation of the function $\mu(s, u, \omega)=\arctan \Phi_{\omega}(s, u, \omega)$, the supremum and infimum are again taken over $s \in[-\pi, \pi],|u| \leqq P,|\omega| \leqq R$. Then

$$
\left\|\mathrm{e}^{H(\mu)}\right\|_{x},\left\|\mathrm{e}^{-H(\mu)}\right\|_{\kappa} \leqq\left(\frac{2 \pi .}{\cos x \gamma_{P . R}}\right)^{1 / x},
$$

according to the well-known Zygmund lemma (cf. [22, 18]). Therefore,

$$
\|\varphi\|_{p} \leqq(2 \pi)^{2 / x}\left\|g_{0}\right\|_{e}\left\{(1 / 2)+A_{r}\left(\cos x \gamma_{P, R}\right)^{-2 / x}\right\},
$$

and because of

$$
\left|k_{1}\right| \leqq \int_{-\pi}^{\pi}|\varphi(s, \xi, \dot{\eta})| d s \leqq(2 \pi)^{1 / q}\|\varphi\|_{p}
$$

we have the estimation

$$
\begin{aligned}
& \left|u_{1}(s)\right| \leqq 2(2 \pi)^{1 / q}\|\varphi\|_{p} \\
& \therefore \quad \leqq(2 \pi)^{1 / \sigma}\left\|g_{0}\right\|_{e}\left\{1+2 A_{r}\left(\cos x \gamma_{P, R}\right)^{-2 / x}\right\},
\end{aligned}
$$

taking into account that $1 / \sigma=1 / q+2 / x$. Moreover,

$$
\begin{aligned}
\left|u_{1}\left(s_{1}\right)-u_{1}\left(s_{2}\right)\right| & \leqq\left|s_{1}-s_{2}\right|^{1 / q}\|\varphi\|_{p} \\
- & \leqq\left|s_{1}-s_{2}\right|^{2}(2 \pi)^{2 / x} \cdot\left\|g_{0}\right\|_{e}\left\{(1 / 2)+A_{r}\left(\cos x \gamma_{P . R}\right)^{-2 / x}\right\} .
\end{aligned}
$$

Finally, we put $P_{0}=R$ and make the Assumption $\mathrm{D}$ that there exist $P, R>0$ with $R=2(2 \pi)^{1 / q} R_{0}, P=2 \pi R+K\left(R, R_{0}\right)$ such thàt

$$
R_{0} \geqq C_{P, R} \equiv(2 \pi)^{2 / x}\left[M+v_{P, R}+R \beta_{P, R}\right]\left\{1+2 A_{r}\left(\cos x \gamma_{P, R}^{\prime}\right)^{-2 / x}\right\},
$$

where as above $1<p<\dot{<}, x=2 p \varrho /[\varrho-p], r=x[\varrho-p] /[\varrho+p], A_{r}$ the M. Riesz constant, and $\nu_{P, R}, \beta_{P, R}, \gamma_{P, R}$ are defined by (39), (40), (41), respectively. Then, on account of the estimations (37) and (44), (45) with (38), the operator $N$ maps the convex compact subset $\mathfrak{\Re}$ of $C(\Gamma) \times C_{0}(\Gamma)$ into itself. Besides, the operator $N: \mathbb{\Re} \rightarrow \mathfrak{R}$ is continuous in the maximum norm of uniform convergence. This is obvious for $N_{1}$ and can be shown for $N_{2}$ like for the corresponding operator in [18].

The Schauder fixed point theorem applied to the equations (29), (30) in $\mathfrak{R}$ now yields the existence of a solution $u(z)$ to Problem $\mathrm{P}$. The boundary values $u(s)$ of the solution $u$ and $u_{1}(s)$ of $\partial u / \partial s$ are lying in $\AA_{1}$ and $\AA_{2}$, respectively: Therefore, $u(z)$ has Hölder continuous partial derivatives in $\bar{G}$, viz. $u(z) \in C^{1 / \lambda}(\bar{G})$ :

Theorem 1: Under. Assumptions A-D Problem $\mathbf{P}$ has a solution $u(z) \in C^{1, \lambda}(\bar{G})$ with the Hölder exponent $\lambda=1-(1 / p), 1<p<\varrho$. 
Corollary: Assumption $D$ is fulfilled for arbitrary. $M$ with a sufficiently large $R>0$ if

$$
\begin{aligned}
& y=\sup \left\|\Phi_{s}(s, u, \omega)\right\|_{e}<\infty, \quad \beta=\sup \left\|\Phi_{s}(s, u, \omega)\right\|_{e}<\infty, \\
& 2 \gamma=\sup \mu(s, u, \omega)-\inf \mu(s, u, \omega)<\pi / x ;
\end{aligned}
$$

the suprenum and infimum are taken over $s \in[-\pi, \pi], u \in \mathbf{R} ; \omega \in \mathbf{R}$, and

$$
2(2 \pi)^{1 / \sigma} \beta\left\{1+2 \dot{A}_{r}(\cos x y)^{-2 / x}\right\}<1 .
$$

Especially, for $\varrho=\infty$ with $\sigma=1, \varkappa=r=2 p$ and $p$ sufficiently near to 1 the condition

$$
4 \pi \beta\left\{1+2(\cos 2 \gamma)^{-1}\right\}<1
$$

insures the existence of a solution to Problem $P$ if the inequalities (2), (3) are-fulfilled uniformly in $u, \omega \in \mathbf{R}$, the oscillation $2 \gamma$ of $\mu(s, u, \omega)=\arctan \Phi_{\omega}(s, u, \omega)$ taken over in $[-\pi, \pi] \times \mathbf{R} \times \mathbf{R}$ is smaller than $\pi / 2$ and Assumption $B$ is satisfied for every $\eta \in C_{0}(\Gamma)$.

- Remark: Using the elementary inequality

$$
|\alpha(s) \cdot a+\beta(s) \cdot b| \leqq \sqrt{a^{2}+b^{2}}
$$

together with Minkowski's inequality in the estimation $(*)$ of $\|\varphi\|_{p}$ we obtain the slightly smaller expression

$$
C_{P, R}=2(2 \pi)^{2 / x}\left[M+v_{P, R}^{\jmath}+R \beta_{P, R}\right]\left\{1+A_{r}^{2}\left(\cos x \gamma_{P, R}\right)^{-4 / x}\right\}^{1 / 2}
$$

in Assumption $\mathrm{D}$ for $p \geqq 2$.

\section{Special cases}

We give some examples taking $\varrho=\infty$ so that the criterion $(50)$ applies.

Example $1:$ Let be $\Phi(s, u, \omega)=c u+\Psi(\omega)$, i.e.

$$
\partial u / \partial r+i c u,+\Psi(\partial u / \partial s)=f(s) \text { on } \Gamma
$$

with a constant $c$ and a continuously differentiable function $\Psi(\omega)$. Then the condition $(50)$ reads

$$
4 \pi|c|\left\{1+2(\cos 2 \gamma)^{-1}\right\}<1
$$

with $2 \gamma=\sup _{\omega \in \mathbb{R}}\left[\arctan \Psi^{\prime}(\omega)\right]-\inf _{\omega \in \mathbf{R}}\left[\arctan \Psi^{\prime}(\omega)\right]<\pi / 2$ which is fulfilled for sufficiently small $|c|$ if $0<i_{1} \leqq \Psi^{\prime}(\omega)<\infty$ or $0 \leqq \Psi^{\prime}(\omega) \leqq l_{2}<\infty$, for instance. Further, $c$ must be different from zero to insure the solvability of the corresponding equation (32) for the parameter $k$.

The condition (52) is very restrictive for the-constant $c$. It always demands that $|c|<\cdot(12 \pi)^{-1}$, whereas in the limit case $\Psi \equiv 0$ existeñce of a solution is present for all $c \neq 0,-1,-2, \ldots$ We $e^{i}$ therefore apply. another method for positive values $c$ introducing the new unknown function

$$
\text { - } U_{0}(z)=r u_{r}(z)+c u(z) \text {. }
$$

With $u$ also $U_{0}$ is a regular harmonic function in $G$ which is continuous in $\bar{G}$ if $u \in C^{\mathbf{l}}(\bar{G})$. Moreover, $u=P\left[U_{0}\right]$ is uniquely determined by, $U_{0}$ and the conjugate 
harmonic function $V_{0}$ to $U_{0}$ is given by $V_{0}(z)=-u_{s}(z)+c v(z)$, where $v$ is the conjugate harmonic function to $u$. The conjugate functions $v, V_{0}$ are normalized by $V_{0}(0)=v(0)=0$ in the origin such that $v=-H(u)=-H\left(P\left[U_{0}\right]\right)$ and $V_{0}=-H\left(U_{0}\right)$ with $H$ the Hilbert operator (18).

The boundary condition (51) now writes

$$
U_{0}+\Psi\left(c v-\dot{V}_{0}\right)=f(s) \text { on } \Gamma
$$

which has the form of a nonlinear Riemann-Hilbert condition for the holomorphic, function $W_{0}(z)=U_{0}(z)+i V_{0}(z)$. Like in our paper [18] we replace (54) by the differentiated condition

$$
\frac{\partial U_{0}}{\partial s}-\dot{\psi^{\prime}}\left(c v-V_{0}\right) \frac{\partial U_{0}}{\partial r_{-}}+c \Psi^{\prime}\left(c v-V_{0}\right)\left[U_{0}-c u\right]=f^{\prime}(s) \text { on } \Gamma
$$

together with the integral condition

$$
\int_{-\pi}^{\pi} U_{0} d s+\int_{-\pi}^{\pi} \Psi\left(c v-V_{0}\right) d s=\int_{-\pi}^{\pi} f(s) d s
$$

From $(\tilde{5} \tilde{5})$ it follows that

$$
U_{0}\left(\mathrm{e}^{i s}\right)=k+\int_{0}^{s} \varphi(\sigma) d \sigma
$$

with

$$
\varphi(s)=\beta(s) h(s) \div \alpha(s) \mathrm{e}^{-H(\mu)(s)} H\left\{\mathrm{e}^{H(\mu)} h\right\},
$$

where

$$
\begin{aligned}
& \alpha(s)=\Psi^{\prime} / \sqrt{1+\Psi^{\prime 2}}, \quad \beta(s)=1 / \sqrt{1+\Psi^{\prime 2}} \\
& \mu(s)=\arctan \Psi^{\prime}, \quad h(s)=g(s) / \sqrt{1+\Psi^{\prime 2}}
\end{aligned}
$$

and

$$
g(s)=f^{\prime}(s)-c \Psi^{\prime}\left(c v-V_{0}\right)\left[U_{0}-c u\right]
$$

has to fulfil the orthogonality condition

$$
\int_{-\pi}^{\pi} \gamma(s) g(s) d s=0
$$

with the nonnegative function

$$
\gamma(s)=\mathrm{e}^{I(\mu)(s)} / \sqrt{1+\Psi^{\prime 2}}
$$

The constant $k$ in (57) is determined by substituting $U_{0}$ into (56).

The investigation of the integral equation (57) for $U_{0}$ can be performed like for the corresponding integral equation in [18], i.e. like in the above proof of Theorem 1 , where now. only the elementary inequalities $|\alpha(s)|,|\beta(s)| \leqq 1$ in the estimation for $\|\varphi\|_{p}$ are used. Restricting ourselves to the case $\varrho=\infty$ again, we have the estimation

with

$$
\|g\|_{\infty} \leqq\left\|f^{\prime}\right\|_{\infty}+2 c \delta\left\|U_{0}\right\|_{\infty}
$$

$$
\delta=\sup _{\omega \in \mathbb{R}}\left|\Psi^{\prime}(\omega)\right|<\infty \quad \text { (by assumption) }
$$


for the function (61) because

$$
\max _{r}|c u| \leqq \max _{r}\left|c u+u_{r}\right|=\max _{r}\left|U_{0}\right|
$$

in virtue of the maximum-minimum property for harmonic functions. In the analogon of the condition (50) the constant $\beta$ can therefore put equal to $2 c \delta$ and we obtain the condition

$$
16 \pi i \delta\left\{1+(\cos 2 \gamma)^{-1}\right\}<1
$$

for positive constant $c$. In the limit case $\Psi \equiv 0$ this condition is fulfilled for all $c>0$.

Example 2: Let be $\Phi(s, u, \omega)=X(u) \Psi^{\prime}(\omega)$, i.e.

$$
\partial u / \partial r+X(u) \Psi^{\prime}(\partial u / \partial s)=f(s) \text { on } \Gamma
$$

with continuously differentiable functions $\Psi(\omega)$ and $X(u)$. Further shall be

$$
\beta_{1}=\sup _{\omega \in \mathbf{R}}|\Psi(\omega)|<\infty, \quad \beta_{2}=\sup _{u \in \mathbf{R}}\left|X^{\prime}(u)\right|<\infty
$$

and

$$
2 \gamma=\sup \left[\arctan \left(X(u)_{i} \Psi^{\prime}(\omega)\right)\right]-\inf \left[\arctan \left(X(u) \Psi^{\prime}(\omega)\right)\right]<\pi / 2,
$$

where supremum and infimum are taken over $u \in \mathbf{R}, \omega \in \mathbf{R}$. Then the condition (50) reads

$$
4 \pi \beta_{1} \beta_{2}\left\{1+2(\cos 2 \gamma)^{-1}\right\}^{\prime}<1
$$

Moreover, Assumption $B$ has to be fulfilled.

In particular, for bounded monotonically increasing function $X(u)$ and' bounded nondecreasing function $\Psi(\omega)$ with

and

$$
\begin{aligned}
& 0 \leqq l_{1}=\lim _{u \rightarrow-\infty} X(u), \quad \lim _{u \rightarrow+\infty} X(u)=L_{1}<\infty \\
& 0<m_{1}=\lim _{\omega \rightarrow-\infty} \Psi(\omega), \quad \lim _{\omega \rightarrow+\infty} \Psi(\omega)=M_{1}<\infty
\end{aligned}
$$

$$
0 \leqq l_{2} \leqq X^{\prime}(u) \leqq L_{2}<\infty, \quad 0 \leqq m_{2} \leqq \psi^{\prime}(\omega) \leqq M_{2}<\infty,
$$

the condition (70) takes the form

$$
\left.4 \pi M_{1} L_{2}\{1+2] \sqrt{1+\left[\frac{L_{1} M_{2}-l_{1} m_{2}}{1+l_{1} L_{1} m_{2} M_{2}}\right]^{2}}\right\}<1 .
$$

And Assumption B is fulfilled if $l_{1} M_{1}<L_{1} m_{1}$ and the right-hand side $f(s)$. satisfies the Landesman-Lazer condition

$$
l_{1} M_{1}<\frac{1}{2 \pi} \int_{-\pi}^{\pi} f(s) d s<L_{1} m_{1} .
$$

Example 3: If $\Phi=\Phi(s, \omega)$ does not depend on $u$, only the equation (30) with the operator $N_{2}$ for the unknown function $\eta$ has to be considered. A solution $u$ of Problem $\mathrm{P}$ exists only if the solution $u_{1}$ of this equation satisfies the condition

$$
\int_{-\pi}^{\pi} \Phi\left(s, u_{1}(s)\right) d \bar{s}=\int_{-\pi}^{\pi} f(s) d s
$$

the solution $u$ is then determined up to an arbitrary additive constant $k$ in (29). 
Since $u_{1}$ is determined by $f^{\prime}(s)$ only, the boundary condition (1) should be modified in the following way

$$
\partial u / \partial r_{i}+\Phi(s, \partial u / \partial s)=f(s)+\lambda \text { on } \Gamma
$$

where $\lambda$ is an arbitrarily variable constant which will be determined by

$$
2 \pi \lambda=\int_{-\pi}^{\pi} \Phi\left(s, u_{1}(s)\right) d s-\int_{-\pi}^{\pi} f(s) d s
$$

after solving the equation (30). Besides, $u$ may be fixed through $u_{1}$ prescribing the value of $k=u(1)$ or

$$
u(0)=\frac{1}{2 \pi} \int_{-\pi}^{\pi} u\left(e^{i s}\right) d s
$$

The existence of a solution $u_{1}$ to the equation (30) is insured if the corresponding Assumptions $\mathrm{A}$ and $\mathrm{C}$ above and the following modified Assumption . D is fulfilled (cf. also [18])':

There exists $R>0$ with $R=2(2 \pi)^{1 / q} R_{0}$ such that

where

$$
R_{0} \geqq C_{R} \equiv(2 \pi)^{2 / x}\left[M+\nu_{R}\right]\left\{1+2 A_{r}\left(\cos x \gamma_{R}\right)^{-2 / x}\right\}
$$

and

$$
v_{R}=\sup \left\|\Phi_{s}(s, \omega)\right\|_{e}<\infty
$$

$$
2 \gamma_{R}=\sup \left[\arctan \Phi_{\omega}(s, \omega)\right]-\inf \left[\arctan \Phi_{\omega}(s, \omega)\right]<\pi / x,
$$

the supremum and infimum are taken over $s \in[-\pi, \pi] ;|\omega| \leqq R$. :

\section{$\S 5$. The quasilinear case}

In the quasilinear case $\Phi(s, u, \omega)=\Psi(s, u)+\omega X(u)$, i.e.

$$
\partial u / \partial r+X(u) \partial u / \partial s+\Psi(s, u)=f(s) \text { on } \Gamma
$$

with Hölder continuous functions $X(u), \Psi(s, u)$, and $f(s)$ we can the above method. apply directly to the boundary condition (82) without differentiate it before. As above this leads to the fixed point problem for the operator $N$ defined by: (cf. also [18])

$$
u(s)=(N \xi)(s)=k+\int_{0}^{s} \varphi(\sigma, \xi) d \sigma
$$

with

where

$$
\varphi(s, \xi)=\beta(\xi) h(s, \xi)+\alpha(\xi) \mathrm{e}^{-H(\mu)(s)} H\left\{\mathrm{e}^{H(\mu)} h\right\}(s),
$$

$$
\begin{aligned}
& \alpha(\xi)=1 / \sqrt{1+X^{2}(\xi)}, \quad \beta(\xi)=X(\xi) / \sqrt{1+X^{2}(\xi)} \\
& \mu(\xi)=\arctan X(\xi), \quad h(s ; \xi)=g_{0}(s ; \xi) / \sqrt{1+X^{2}(\xi)}
\end{aligned}
$$


and

$$
\begin{aligned}
& g_{0}(s, \xi)=g(s, \xi)-m_{0}[\xi], \\
& g(s, \xi)=f(s)-\Psi(s, \xi), \\
& m_{0}[\xi]=\int_{-\pi}^{\pi} g(s, \xi) \gamma(s, \xi) d s / \int_{-\pi}^{\pi} \gamma(s, \xi) d s, \\
& \gamma(s, \xi)=\mathrm{e}^{H(\mu)(s)} / \sqrt{1+X^{2}(\xi)} .
\end{aligned}
$$

Here the constant $k$ in (83) is to be determined in fulfilling the integral condition

$$
\left.\int_{-\pi}^{\pi} \Psi\left(s, k+\int_{0}^{s} \varphi(\sigma, \xi) d \sigma\right)\right) d s=\int_{-\pi}^{\pi} f(s) d s .
$$

We assume that

$$
2 \gamma=\sup [\arctan \bar{X}(u)]-\inf [\arctan X(u)]<\pi / 2,
$$

the supremum and infimum are taken over $\ddot{u} \in \mathbf{R}$; and

$$
|\Psi(s, u)| \leqq c_{1}+c_{2}|u|^{\delta}, \quad 0 \leqq \delta<1,
$$

for all $u \in \mathbf{R}$ with positive constants $c_{1}, c_{2}$. Furthermore, the equation (91) for $k \in \mathbf{R}$ shall have a root $k=k[\xi]$ for any $\xi \in \bar{C}(\Gamma)$ which depends continuously upon $\xi$ and satisfies the estimation

$$
\therefore|k[\xi]| \leqq K_{1}+K_{2}^{\prime} R^{\nu} \equiv K(R), \quad 0 \leqq v<1,
$$

for all $\xi \in C(\Gamma)$ with $|\xi(s)| \leqq R, R>\dot{0}$, and uniform positive constants $K_{1}, K_{2}$. This - is especially fulfilled with $\delta<v<1$ if the (continuous) function $\Psi(s, u)$ is strictly monotone with respect to $u$ for any $s \in[-\pi, \pi]$, there exist the (finite or identically infinite) limit functions

$$
\lim _{u \rightarrow \pm \infty} \Psi(s, u)=\Psi_{ \pm}(s) \text { uniformly in } s \in[-\pi, \pi]
$$

and $\cdot \int_{-\pi}^{\pi} f(s) d s$ lies between the values $\Psi_{ \pm}=\int_{-\pi}^{\pi} \Psi_{ \pm}(s) d s$. Namely, because of the assumptions (92) and (93), there holds an' estimation of the form

$$
\int_{-\pi}^{\pi}|\varphi(s, \xi)| d s \leqq L_{1}+\dot{L}_{2} R^{\delta}
$$

for all $\xi \in C(\Gamma)$.with $|\xi(s)| \leqq R$ and uniform positive constants $L_{1}, L_{2}$ as can be shown like above.

Now we consider the operator $N$ on the convex compact set $\mathfrak{R}$ of $C(\Gamma)$ defined by

$$
\mathfrak{N}=\left\{\xi \in C(\Gamma):|\xi(s)| \leqq R,\left|\xi\left(s_{1}\right)-\xi\left(s_{2}\right)\right| \leqq R_{0}\left|s_{1}-s_{2}\right|^{2}\right\}
$$

with $\lambda=1-(1 / p), 1<p<\pi /(4 \gamma)$, and sufficiently large positive constant $\dot{R}$ such that $(2 \pi)^{\lambda} R_{0}=R-K(R)=R-K_{1}-K_{2} R^{\nu}>0$ and

$$
R_{0} \geqq C_{R}=(2 \pi)^{1 / p}\left[M+c_{1}+c_{2} R^{d}\right]\left\{1+2 A_{2 p}(\cos 2 p \gamma)^{-1 / p}\right\}
$$

with $M=\max _{\Gamma}|f(s)|$ and $A$, the M. Riesz constant. Then like in [18] Schauder's fixed point theorem yields the existence of a fixed point $u \in \mathfrak{R}$ for the operator $N$. 
Moreover, because of $\partial u / \partial s=\varphi(s, u)$ the corresponding solution $u(z)$ to Problem $\mathrm{P}$ has Hölder continuous partial derivatives in $\bar{G}$. Using the Lipschitz continuity of $u$ in (84) the corresponding Hölder exponent is seen to be the minimum of the Hölder exponents of $f, \Psi, X$.

Theorem 2: If the assumptions (92), (93) are fulfilled and there exists a continunus solution $k[\xi]$ of (91) with (94) the boundary value problem (82) with Hölder continuous functions $X(u), \Psi(s, u)$, and $f(s)$ has a solution $u(z) \in C^{1 . \varepsilon}(\bar{G})$, where $\varepsilon$ is the minimum of the Hölder exponents of $f, \Psi, X$.

If the function $\Psi(s, u)$ in (82) is strictly increasing in $u$, existence of solutions to this problem can also be proved for functions $\Psi$ with linear and superlinear growth in $u^{r}$ using results of SchLEIFF [16] for the corresponding semilinear boundary condition.

In the boundary condition

$$
\partial u / \partial r+X(s, u) \partial u / \partial s+\Psi(s, u)=f(s) \text { on } \Gamma
$$

the functions $X(s, u), \Psi(s, u), f(s)$ shall be Hölder continuous and moreover, $\Psi(s, u)$ shall possess a Hölder continuous derivative $\Psi_{u}(s, u)>0$ for all $s \in[\dot{-} \pi, \pi], u \in \mathbf{R}$. Further we assume that

$$
\Psi_{-}(s)<f(s)<\Psi_{+}(s) \text {. for } s \in[-\pi, \pi],
$$

where $\Psi_{ \pm}(s)=\lim _{u \rightarrow \pm \infty} \Psi^{\prime}(s, u)$

Then, according to Theorem 2 of [16: Part 2] for any'Hölder continuous function $\xi=\xi(s)$ the auxiliary problem

$$
\partial u / \partial \dot{r}+X(s, \xi) \partial u \dot{u} \partial s+\Psi(s, u) \stackrel{\text { ' }}{=} f(s) \text { on } \Gamma
$$

has a unique solution $\overrightarrow{u(z)}$ with Hölder continuous partial derivatives in $\bar{G}$. Moreover, $u(z)$ satisfies the estimation,

$$
\eta_{1} \equiv \min _{r} \eta(s) \leqq u(z) \leqq \max _{\Gamma} \eta(s) \equiv \eta_{2}, \quad z \in \bar{G}
$$

where the continuous function $\eta(s)$ is the solution $u$ to the equation

$$
\Psi(s, u)=f(s), \quad s \in[-\pi, \pi] .
$$

We consider the problem (101) for $\xi \in \mathfrak{N}$ with $\mathfrak{R}$ defined by

$$
\mathfrak{R}=\left\{\xi \in C(T): \eta_{1} \leqq \xi(s) \leqq \eta_{2}, \quad\left|\xi\left(s_{1}\right)-\xi\left(s_{2}\right)\right| \leqq R_{0}\left|s_{1}-s_{2}\right|^{\lambda}\right\},
$$

where $\lambda=1-(1 / p), 1<p<\pi /(4 \gamma)$. The solution $u$ of (101) depends continuously on $\xi \in \mathfrak{R}$ in the maximum norm topology such that the operator $u=N \xi$ of the boundary values $u(s)$ of $u$ is a continuous operator from into $C(\Gamma)$.

Namely, let $\xi_{n} \in \mathfrak{R}$ converge uniformly to $\xi_{0} \in \mathfrak{R}$ and let $u_{n}, u_{0}$ be the corresponding solutions of (101), respectively. The difference function $U_{n}=u_{n}-u_{0}$ satisfies the boundary condition.

$$
\partial U_{n} / \partial r+X\left(s, \xi_{n}\right) \partial U_{n}^{\prime} / \partial s+\Psi\left(s, U_{n}+u_{0}\right)=G_{n}(s) \text { on } \Gamma
$$

with the Hölder continuous function

$$
G_{n}(s)=\Psi\left(s, u_{0}\right)+\left[X\left(s, \xi_{0}\right)-X\left(s, \xi_{n}\right)\right] \partial u_{0} / \partial s .
$$


Again by Theorem 2 of [16: Part 2] there holds the estimation

$$
\min _{\Gamma} \eta_{n}(s) \leqq U_{n}(s) \leqq \max _{\Gamma} \eta_{n}(s), \quad s \in[-\pi, \pi],
$$

for the boundary values of $U_{n}$ on $\Gamma$, where $\eta_{n}(s)$ is the unique solution of the equation

$$
\Psi\left(s, \eta_{n}(s)+u_{0}(s)\right)=G_{n}(s), \quad s \in[-\pi ; \pi] .
$$

But $G_{n}(s)$ converges uniformly to $\Psi\left(s, u_{0}(s)\right)$ as $n \rightarrow \infty$ such that $\eta_{n}(s)$ converges uniformly to zero. Due to $(106)$ then also $U_{n}(s)$ converges uniformly to zero.

Further, analogously to (83) one obtains the following expression for $u=N \xi$ :

$$
u(s)=k+\int_{0}^{s} \varphi(\sigma, \xi, u) d \sigma
$$

with $k=k[\xi]$ the value of $u(z)$ for $t=1$ and $\varphi(s, \xi, u)$ is given by (84), where in the formulas $(84)-(90) X(s, \xi)$ instead of $X(\xi), \Psi(s, u)$ instead of $\Psi(s, \xi)$, and $g(s, u)$ instead of $g_{0}(s, \xi)$ is to be written. As just proved, $u(z)$ depends continuously upon $\xi$, therefore also $k=k[\xi]$.

Finally, we assume that.

$$
2 \gamma^{\prime}=\sup [\arctan X(s, u)]-\inf [\arctan X(s, u)]<\pi / 2,
$$

the supremum and infimum are taken over $s \in[-\pi, \pi], \eta_{1} \leqq u \leqq \eta_{2}$. Then there holds the estimation

$$
\|\varphi\|_{p} \leqq(2 \pi)^{1 / p}\left\{M+M_{0}\right\}\left\{(1 / 2)+A_{2 p}(\cos 2 p \gamma)^{-1 / p}\right\} \equiv C_{0}
$$

fòr any $\xi \in \mathfrak{R}$, where $1<p<\pi /(4 \gamma), M=\max _{\Gamma}|f(s)|$,

$$
M_{0}=\max _{\Gamma} \max \left[\Psi\left(s, \eta_{2}\right),-\Psi\left(s, \eta_{1}\right)\right]
$$

and $A_{r}$ the' M. Riesz constant.

Taking the constant $R_{0}$ in (104) equal to $C_{0}$ and applying the Schauder fixed point theorem to the operator $N$ on $\Re$, we obtain the existence of a fixed point of $N$, i.e. the existence of a solution $u$ to the boundary value problem (99).

Theorem 3: Under the assumptions (100) and (109) the boundary value problem (99) with Hölder continuous functions $X(s, u), \Psi(s, u), f(s)$, where. $\Psi(s, u)$ possesses a Hölder continuous derivative $\Psi_{u}(s, u)>0$, has a solution $u(z) \in C^{1, \varepsilon}(\bar{G})$, where' $\varepsilon$ is the minimum of the Hölder exponents of $f, \Psi, X$.

Finally, we briefly deal with the boundary condition of the form

$$
\partial u / \partial r+\partial / \partial s[\Phi(s, u)]=f(s) \text { on } \Gamma
$$

with Hölder continuous function $f(s)$ satisfying the necessary solvability condition

$$
\int_{-\pi}^{\pi} f(s) d s=0
$$

and Hölder continuously differentiable function $\Phi(s, u)$ with respect to $s$ and $u$.

Denoting by $v(z)$ the conjugate harmonic function to $u(z)$ normalized by $v(1)=0$ or $v(0)=0$, respectively, the condition (111) is equivalent to the Riemann-Hilbert condition

$$
v+\Phi(s, u)=\bar{k}+F(s) \text { on } \Gamma
$$


with $F(s)=\int_{0}^{s} f(\sigma) d \sigma$ and an arbitrary constant $\bar{k} \in \mathbf{R}$ together with the additional
condition

$$
v(\mathbf{1})=0
$$

or

$$
v(0)=0
$$

respectively.

The Riemann-Hilbert problems (113) with (114) or (115) have been considered in our papers $[18,19]$. In case of the additional condition (114) let $k \in \mathbf{R}$ be an arbitrary constant and put $\vec{k}=\Phi(0, k)$. Then (114) can be replaced by the ádditional condition

$$
u(1)=k
$$

to the boundary condition

$$
v+\Phi(s, u)=\Phi(0, k)+F(s) \text { on' } \Gamma \text {. }
$$

To this problem Theorem. 2 of [18] with $\varrho=\infty$ can be applied.

For strictly monotone function $\Phi(s, u)$ in $u$ also Theorem 4 of [18] with $\varrho=\infty$ may be applied directly to the problem (113) with (115), where the Landesman-Lazer type condition in this theorem can be fulfilled by a suitable choice of the parameter $\vec{k}$.

\section{REFERENCES}

[1] Amanx, H.: Nonlinear operators in ordered Banach spaces and some applications to nonlinear boundary value problems. In: Nonlinear Operators and the Calculus of Variations, Bruxelles 1975 (Lecture Notes Math. 543). Springer-Verlag: Berlin-Heidelberg New York 1976, pp. 1-55.

[2] Amary, H.: Nonlinear elliptic equations with nonlinear boundary conditions. In: New Developments in Differential Equations (ed. by W: Eckhaus). North-Holland Publ. Comp., Amsterdam 1976, p. 43-63.

[3] Carlemax, T.: Uber eine nichtlineare Randwertaufgabe bei dé Gleichung- $\Delta u=0$. Math. Zeitschrift 93 (1921), 35-43.

[4] Cusmino, J. M.: Nonlinear Steklov problems on the unit circle. J. Math. Anal. Appl. 38 (1972), $766-783$.

[5] ГАхов, Ф. Д.: Краевые задачи. Гос. изд-во физ.-мат. лит.: Москва 1963.

[6] INKMaNN, F.: Existence and multiplicity theorems for semi-linear elliptic equations with nonlinear boundary conditions. Indiana Univ. Math. J. 31 (1982), 213-221:

[7]: Klingelmöfer, K.: Uber nichtlineare Randwertaufgaben der Potentialtheorie I, II. Mitteil. math. Sem. Gießen 76 (1967), 1-70; 79 (1968); $1-27$.

[8] Klingelföfer, K.: Nonlinear harmonic boundary value problems I. Arch. Rat. Mcch. Añal. 31 (1968), 364-371.

[9] KLINGelhöfer, K:: Nonlinear harmonic boundary value problems II. (modified Ham. merstein integral equations). J. Math. Anal. Appl. 25 (1969), 592-606.

[10] Klingflö̈FER, K.: Modified Hammerstein integral equations and nonlinear harmonic boundary value problems. J. Math. Anal. Appl. 28 (1969), $77-87$.

[11] KLINGELHöFER, K.: Nonlinear boundary value problems with simple eigenvalue of the linear part. Arch. Rat. Mech. Anal. 37 (1970), 381 - 398.

[12] Maruhn, K.: Úbèr einige Klassen nichtlinearer Randwertaufgaben der Potentialtheorie. Math. Z. 51 (1949), 36-60.

[13] Muschelisćnwidi, N. I.: Singuläre Integralgleichungen. Randwertprobleme der Fúnktionentheorie und einige Anwendungen auf die mathematische Physik (Math. Lehrbücher u. Monographien: Abt. 2, Bd. 20). Akademie-Verlag: Berlin 1965. 
[14] Pogonzelski, W.: Integral Equations and their Applications, Vol. I (International Series of Monographs in Pure and Applied Mathematics: Vol. 88). Pergamon Press: Oxford, and PWN: Warszawa 1966.

[15] Rotнe, F.: Sölutions for systems of nonlinear elliptic equations with nonlinear boundary

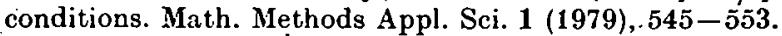

[16] SchleifF, M.: Utber einige nichtlineare Verallgemeinerungen des Randwertproblems von Poincaré, 1. und 2. Teil. Wiss. Z. Univ. Halle 19 (1970), 87-93 und 95-100.

[17] Whotiuer, P.: Existence of a minimal solution and a maximal solution of nonlinear elliptic boundary value problems. Indiana Univ. Math. J. $99(1980), \overline{4} 55-462$.

[18] Wolfersporf, L. v.: A class of nonlinear Riemann-Hilbert problems for holomorphic functions. Math. Nachr. 116 (1984), 89-107.

[19] WOLFERSDORF, L. v: : Landesman-Lazer's type boundary value problems for holomorphic functions. Math. Nachr. 114 (1983), 181-189.

[20] Wolska-Bochesek, J.: Un problème aùx limites à dérivée tangéntielle pour l'equation : du type elliptique. Ann. Polon. Math. 4 (1958), 257-287:

[21] Wolska-Bochenek, J.: Problème non-linéaire à dérivée oblique. Ann. Polon. Math. 9 (1961), $253-264$.

[22] Zygmond, A.: Trigonometrical Series (Monografie mathematýczne: T. 5). Univ.: Warszawa and Lwów: 1935.

Manuskripteingang: 07.03 .1983

\section{VERFASSER:}

Prof. Dr. Lothar von Wolfersdorf

Sektion Mathematik der Bergakademie Freiberg

DDR - 9200 Freiberg, Akademiestr. 6 